\title{
Loops on spheres having a compact-free inner mapping group
}

\author{
Ágota Figula and Karl Strambach
}

\begin{abstract}
We prove that any topological loop homeomorphic to a sphere or to a real projective space and having a compact-free Lie group as the inner mapping group is homeomorphic to the circle. Moreover, we classify the differentiable 1-dimensional compact loops explicitly using the theory of Fourier series.
\end{abstract}

2000 Mathematics Subject Classification: 22A30, 22E99, 20N05, 57S20, 22F30

Key words and phrases: locally compact loops, differentiable loops, multiplications on spheres

\section{Introduction}

The only known proper topological compact connected loops such that the groups $G$ topologically generated by their left translations are locally compact and the stabilizers $H$ of their identities in $G$ have no non-trivial compact subgroups are homeomorphic to the 1-sphere. In [8], [9], [7, [10] it is shown that the differentiable 1-dimensional loops can be classified by pairs of real functions which satisfy a differential inequality containing these functions and their first derivatives. A main goal of this paper is to determine the functions satisfying this inequality explicitly in terms of Fourier series.

If $L$ is a topological loop homeomorphic to a sphere or to a real projective space and having a Lie group $G$ as the group topologically generated by the left translations such that the stabilizer of the identity of $L$ is a compact-free Lie subgroup of $G$, then $L$ is the 1-sphere and $G$ is isomorphic to a finite covering of the group $P S L_{2}(\mathbb{R})$ (cf. Theorem 4).

To decide which sections $\sigma: G / H \rightarrow G$, where $G$ is a Lie group and $H$ is a (closed) subgroup of $G$ containing no normal subgroup $\neq 1$ of $G$ correspond to loops we use systematically a theorem of R. Baer (cf. [3] and [8], Proposition 1.6, p. 18). This statement says that $\sigma$ corresponds 
to a loop if and only if the image $\sigma(G / H)$ is also the image for any section $G / H^{a} \rightarrow G$, where $H=a^{-1} H a$ and $a \in G$. As one of the applications of this we derive in a different way the differential inequality in [8], p. 218 , in which the necessary and sufficient conditions for the existence of 1-dimensional differentiable loops are hidden.

\section{Basic facts in loop theory}

A set $L$ with a binary operation $(x, y) \mapsto x * y: L \times L \rightarrow L$ and an element $e \in L$ such that $e * x=x * e=x$ for all $x \in L$ is called a loop if for any given $a, b \in L$ the equations $a * y=b$ and $x * a=b$ have unique solutions which we denote by $y=a \backslash b$ and $x=b / a$. Every left translation $\lambda_{a}: y \mapsto a * y: L \rightarrow L, a \in L$ is a bijection of $L$ and the set $\Lambda=\left\{\lambda_{a}, a \in L\right\}$ generates a group $G$ such that $\Lambda$ forms a system of representatives for the left cosets $\{x H, x \in G\}$, where $H$ is the stabilizer of $e \in L$ in $G$. Moreover, the elements of $\Lambda$ act on $G / H=\{x H, x \in G\}$ such that for any given cosets $a H$ and $b H$ there exists precisely one left translation $\lambda_{z}$ with $\lambda_{z} a H=b H$.

Conversely, let $G$ be a group, $H$ be a subgroup containing no normal subgroup $\neq 1$ of $G$ and let $\sigma: G / H \rightarrow G$ be a section with $\sigma(H)=1 \in G$ such that the set $\sigma(G / H)$ of representatives for the left cosets of $H$ in $G$ generates $G$ and acts sharply transitively on the space $G / H$ (cf. [8], p. 18). Such a section we call a sharply transitive section. Then the multiplication defined by $x H * y H=\sigma(x H) y H$ on the factor space $G / H$ or by $x * y=\sigma(x y H)$ on $\sigma(G / H)$ yields a loop $L(\sigma)$. The group $G$ is isomorphic to the group generated by the left translations of $L(\sigma)$.

We call the group generated by the mappings $\lambda_{x, y}=\lambda_{x y}^{-1} \lambda_{x} \lambda_{y}: L \rightarrow$ $L$, for all $x, y \in L$, the inner mapping group of the loop $L$ (cf. [8], Definition 1.30, p. 33). According to Lemma 1.31 in [8], p. 33, this group coincides with the stabilizer $H$ of the identity of $L$ in the group generated by the left translations of $L$.

A locally compact loop $L$ is almost topological if it is a locally compact space and the multiplication $*: L \times L \rightarrow L$ is continuous. Moreover, if the maps $(a, b) \mapsto b / a$ and $(a, b) \mapsto a \backslash b$ are continuous then $L$ is a topological loop. An (almost) topological loop $L$ is connected if and only if the group topologically generated by the left translations is connected. We call the loop $L$ strongly almost topological if the group topologically generated by its left translations is locally compact and the corresponding sharply transitive section $\sigma: G / H \rightarrow G$, where $H$ is the stabilizer of $e \in L$ in $G$, is continuous.

If a loop $L$ is a connected differentiable manifold such that the multiplication $*: L \times L \rightarrow L$ is continuously differentiable, then $L$ is an 
almost $\mathcal{C}^{1}$-differentiable loop (cf. Definition 1.24 in [8], p. 31). Moreover, if the mappings $(a, b) \mapsto b / a$ and $(a, b) \mapsto a \backslash b$ are also continuously differentiable, then the loop $L$ is a $\mathcal{C}^{1}$-differentiable loop. If an almost $\mathcal{C}^{1}$-differentiable loop has a Lie group $G$ as the group topologically generated by its left translations, then the sharply transitive section $\sigma: G / H \rightarrow G$ is $\mathcal{C}^{1}$-differentiable. Conversely, any continuous, respectively $\mathcal{C}^{1}$-differentiable sharply transitive section $\sigma: G / H \rightarrow G$ yields an almost topological, respectively an almost $\mathcal{C}^{1}$-differentiable loop.

It is known that for any (almost) topological loop $L$ homeomorphic to a connected topological manifold there exists a universal covering loop $\tilde{L}$ such that the covering mapping $p: \tilde{L} \rightarrow L$ is an epimorphism. The inverse image $p^{-1}(e)=\operatorname{Ker}(p)$ of the identity element $e$ of $L$ is a central discrete subgroup $Z$ of $\tilde{L}$ and it is naturally isomorphic to the fundamental group of $L$. If $Z^{\prime}$ is a subgroup of $Z$, then the factor loop $\tilde{L} / Z^{\prime}$ is a covering loop of $L$ and any covering loop of $L$ is isomorphic to a factor loop $\tilde{L} / Z^{\prime}$ with a suitable subgroup $Z^{\prime}$ (see [5]).

If $L^{\prime}$ is a covering loop of $L$, then Lemma 1.34 in [8], p. 33, clarifies the relation between the group topologically generated by the left translations of $L^{\prime}$ and the group topologically generated by the left translations of $L$ :

Let $L$ be a topological loop homeomorphic to a connected topological manifold. Let the group $G$ topologically generated by the left translations $\lambda_{a}, a \in L$, of $L$ be a Lie group. Let $\tilde{L}$ be the universal covering of $L$ and $Z \subseteq \tilde{L}$ be the fundamental group of $L$. Then the group $\tilde{G}$ topologically generated by the left translations $\tilde{\lambda}_{u}, u \in \tilde{L}$, of $\tilde{L}$ is the covering group of $G$ such that the kernel of the covering mapping $\varphi: \tilde{G} \rightarrow G$ is $Z^{*}=$ $\left\{\tilde{\lambda}_{z}, z \in Z\right\}$ and $Z^{*}$ is isomorphic to $Z$. If we identify $\tilde{L}$ and $L$ with the homogeneous spaces $\tilde{G} / \tilde{H}$ and $G / H$, where $H$ or $\tilde{H}$ is the stabilizer of the identity of $L$ in $G$ or of $\tilde{L}$ in $\tilde{G}$, respectively, then $\varphi(\tilde{H})=H$, $\tilde{H} \cap Z^{*}=\{1\}$, and $\tilde{H}$ is isomorphic to $H$.

\section{Compact topological loops on the 3-dimensional sphere}

Proposition 1. There is no almost topological proper loop L homeomorphic to the 3-sphere $\mathcal{S}_{3}$ or to the 3-dimensional real projective space $\mathcal{P}_{3}$ such that the group $G$ topologically generated by the left translations of $L$ is isomorphic to the group $S L_{2}(\mathbb{C})$ or to the group $P S L_{2}(\mathbb{C})$, respectively.

Proof. We assume that there is an almost topological loop $L$ homeomorphic to $\mathcal{S}_{3}$ such that the group topologically generated by its left 
translations is isomorphic to $G=S L_{2}(\mathbb{C})$. Then there exists a continuous sharply transitive section $\sigma: S L_{2}(\mathbb{C}) / H \rightarrow S L_{2}(\mathbb{C})$, where $H$ is a connected compact-free 3-dimensional subgroup of $S L_{2}(\mathbb{C})$. According to [2], pp. 273-278, there is a one-parameter family of connected compact-free 3-dimensional subgroups $H_{r}, r \in \mathbb{R}$ of $S L_{2}(\mathbb{C})$ such that $H_{r_{1}}$ is conjugate to $H_{r_{2}}$ precisely if $r_{1}=r_{2}$. Hence we may assume that the stabilizer $H$ has one of the folowing shapes

$$
H_{r}=\left\{\left(\begin{array}{cc}
\exp [(r i-1) a] & b \\
0 & \exp [(1-r i) a]
\end{array}\right) ; a \in \mathbb{R}, b \in \mathbb{C}\right\}, r \in \mathbb{R},
$$

(cf. Theorem 1.11 in [8], p. 21). For each $r \in \mathbb{R}$ the section $\sigma_{r}: G / H_{r} \rightarrow$ $G$ corresponding to a loop $L_{r}$ is given by

$\left(\begin{array}{rr}x & y \\ -\bar{y} & \bar{x}\end{array}\right) H_{r} \mapsto\left(\begin{array}{rr}x & y \\ -\bar{y} & \bar{x}\end{array}\right)\left(\begin{array}{cc}\exp [(r i-1) f(x, y)] & g(x, y) \\ 0 & \exp [(1-r i) f(x, y)]\end{array}\right)$,

where $x, y \in \mathbb{C}, x \bar{x}+y \bar{y}=1$ such that $f(x, y): S^{3} \rightarrow \mathbb{R}, g(x, y): S^{3} \rightarrow \mathbb{C}$ are continuous functions with $f(1,0)=0=g(1,0)$. Since $\sigma_{r}$ is a sharply transitive action for each $r \in \mathbb{R}$ the image $\sigma_{r}\left(G / H_{r}\right)$ forms a system of representatives for all cosets $x H_{r}^{\gamma}, \gamma \in G$. This means for all given $c, d \in \mathbb{C}^{2}, c \bar{c}+d \bar{d}=1$ each coset

$$
\left(\begin{array}{rr}
u & v \\
-\bar{v} & \bar{u}
\end{array}\right)\left(\begin{array}{rr}
c & d \\
-\bar{d} & \bar{c}
\end{array}\right) H_{r}\left(\begin{array}{rr}
\bar{c} & -d \\
\bar{d} & c
\end{array}\right)
$$

where $u, v \in \mathbb{C}, u \bar{u}+v \bar{v}=1$, contains precisely one element of $\sigma_{r}\left(G / H_{r}\right)$. This is the case if and only if for all given $c, d, u, v \in \mathbb{C}$ with $u \bar{u}+v \bar{v}=$ $1=c \bar{c}+d \bar{d}$ there exists a unique triple $(x, y, q) \in \mathbb{C}^{3}$ with $x \bar{x}+y \bar{y}=1$ and a real number $m$ such that the following matrix equation holds:

$$
\begin{gathered}
\left(\begin{array}{cc}
\bar{u} \bar{c}-\bar{v} d & -u d-v \bar{c} \\
\bar{v} c+\bar{u} \bar{d} & u c-v \bar{d}
\end{array}\right)\left(\begin{array}{cc}
x & y \\
-\bar{y} & \bar{x}
\end{array}\right)\left(\begin{array}{cc}
\exp [(r i-1) f(x, y)] & g(x, y) \\
0 & \exp [(1-r i) f(x, y)]
\end{array}\right) \\
=\left(\begin{array}{cc}
\exp [(r i-1) m] & q \\
0 & \exp [(1-r i) m]
\end{array}\right)\left(\begin{array}{rr}
\bar{c} & -d \\
\bar{d} & c
\end{array}\right) .
\end{gathered}
$$

The (1,1)- and (2,1)-entry of the matrix equation (1) give the following system $A$ of equations:

$$
\begin{gathered}
{[(\bar{u} x+v \bar{y}) \bar{c}+(u \bar{y}-\bar{v} x) d] \exp [(r i-1) f(x, y)]=\exp [(r i-1) m] \bar{c}+q \bar{d}} \\
{[(\bar{v} x-u \bar{y}) c+(\bar{u} x+v \bar{y}) \bar{d}] \exp [(r i-1) f(x, y)]=\exp [(1-r i) m] \bar{d}}
\end{gathered}
$$

If we take $c$ and $d$ as independent variables the system $A$ yields the following system $B$ of equations:

$$
(\bar{u} x+v \bar{y}) \exp [\operatorname{irf}(x, y)] \exp [-f(x, y)]=\exp (\operatorname{irm}) \exp (-m)
$$




$$
\begin{gathered}
(u \bar{y}-\bar{v} x) \exp [(r i-1) f(x, y)] d=\bar{d} q \\
(\bar{u} x+v \bar{y}) \exp [i r f(x, y)] \exp [-f(x, y)]=\exp (m) \exp (-i r m) .
\end{gathered}
$$

Since equation (5) must be satisfied for all $d \in \mathbb{C}$ we obtain $q=0$. From equation (4) it follows

$$
\bar{u} x+v \bar{y}=\exp (i r m) \exp (-m) \exp [-\operatorname{irf}(x, y)] \exp [f(x, y)] .
$$

Putting (7) into (6) one obtains

$$
\exp (\text { irm }) \exp (-m)=\exp (m) \exp (- \text { irm })
$$

which is equivalent to

$$
\exp [2(i r-1) m]=1
$$

The equation (9) is satisfied if and only if $m=0$. Hence the matrix equation (1) reduces to the matrix equation

$$
\left(\begin{array}{cc}
x & y \\
-\bar{y} & \bar{x}
\end{array}\right)\left(\begin{array}{cc}
\exp [(r i-1) f(x, y)] & g(x, y) \\
0 & \exp [(1-r i) f(x, y)]
\end{array}\right)=\left(\begin{array}{cc}
u & v \\
-\bar{v} & \bar{u}
\end{array}\right) .
$$

and therefore the matrix

$$
M=\left(\begin{array}{cc}
\exp [(r i-1) f(x, y)] & g(x, y) \\
0 & \exp [(1-r i) f(x, y)]
\end{array}\right)
$$

is an element of $S U_{2}(\mathbb{C})$. This is the case if and only if $f(x, y)=0=$ $g(x, y)$ for all $(x, y) \in \mathbb{C}^{2}$ with $x \bar{x}+y \bar{y}=1$. Since for each $r \in \mathbb{R}$ the loop $L_{r}$ is isomorphic to the loop $L_{r}\left(\sigma_{r}\right)$, hence to the group $S U_{2}(\mathbb{C})$, there is no connected almost topological proper loop $L$ homeomorphic to $\mathcal{S}_{3}$ such that the group topologically generated by its left translations is isomorphic to the group $S L_{2}(\mathbb{C})$.

The universal covering of an almost topological proper loop $L$ homeomorphic to the real projective space $\mathcal{P}_{3}$ is an almost topological proper loop $\tilde{L}$ homeomorphic to $\mathcal{S}_{3}$. If the group topologically generated by the left translations of $L$ is isomorphic to $P S L_{2}(\mathbb{C})$ then the group topologically generated by the left translations of $\tilde{L}$ is isomorphic to $S L_{2}(\mathbb{C})$. Since no proper loop $\tilde{L}$ exists the Proposition is proved.

Proposition 2. There is no almost topological proper loop L homeomorphic to the 3-dimensional real projective space $\mathcal{P}_{3}$ or to the 3 -sphere $\mathcal{S}_{3}$ such that the group $G$ topologically generated by the left translations of $L$ is isomorphic to the group $S L_{3}(\mathbb{R})$ or to the universal covering group $\widetilde{S L_{3}(\mathbb{R})}$, respectively. 
Proof. First we assume that there exists an almost topological loop $L$ homeomorphic to $\mathcal{P}_{3}$ such that the group topologically generated by its left translations is isomorphic to $G=S L_{3}(\mathbb{R})$. Then there is a continuous sharply transitive section $\sigma: S L_{3}(\mathbb{R}) / H \rightarrow S L_{3}(\mathbb{R})$, where $H$ is a connected compact-free 5-dimensional subgroup of $S L_{3}(\mathbb{R})$. According to Theorem 2.7, p. 187, in [4] and to Theorem 1.11, p. 21, in [8] we may assume that

$$
H=\left\{\left(\begin{array}{ccc}
a & k & v \\
0 & b & l \\
0 & 0 & (a b)^{-1}
\end{array}\right) ; a>0, b>0, k, l, v \in \mathbb{R}\right\} .
$$

Using Euler angles every element of $\mathrm{SO}_{3}(\mathbb{R})$ can be represented by the following matrix

$$
\begin{gathered}
g(t, u, z):=\left(\begin{array}{rrr}
\cos t & \sin t & 0 \\
-\sin t & \cos t & 0 \\
0 & 0 & 1
\end{array}\right)\left(\begin{array}{rrr}
1 & 0 & 0 \\
0 & \cos z & \sin z \\
0 & -\sin z & \cos z
\end{array}\right)\left(\begin{array}{rrr}
\cos u & \sin u & 0 \\
-\sin u & \cos u & 0 \\
0 & 0 & 1
\end{array}\right)= \\
\left(\begin{array}{ccc}
\cos t \cos u-\sin t \cos z \sin u & \cos t \sin u+\sin t \cos z \cos u & \sin t \sin z \\
-\sin t \cos u-\cos t \cos z \sin u & -\sin t \sin u+\cos t \cos z \cos u & \cos t \sin z \\
\sin z \sin u & -\sin z \cos u & \cos z
\end{array}\right),
\end{gathered}
$$

where $t, u \in[0,2 \pi]$ and $z \in[0, \pi]$.

The section $\sigma: S L_{3}(\mathbb{R}) / H \rightarrow S L_{3}(\mathbb{R})$ is given by

$$
g(t, u, z) H \mapsto g(t, u, z)\left(\begin{array}{ccc}
f_{1}(t, u, z) & f_{2}(t, u, z) & f_{3}(t, u, z) \\
0 & f_{4}(t, u, z) & f_{5}(t, u, z) \\
0 & 0 & f_{1}^{-1}(t, u, z) f_{4}^{-1}(t, u, z)
\end{array}\right),
$$

where $t, u \in[0,2 \pi], z \in[0, \pi]$ and $f_{i}(t, u, z):[0,2 \pi] \times[0,2 \pi] \times[0, \pi] \rightarrow$ $\mathbb{R}$ are continuous functions such that for $i \in\{1,4\}$ the functions $f_{i}$ are positive with $f_{i}(0,0,0)=1$ and for $j=\{2,3,5\}$ the functions $f_{j}(t, u, z)$ satisfy that $f_{j}(0,0,0)=0$. As $\sigma$ is sharply transitive the image $\sigma\left(S L_{3}(\mathbb{R}) / H\right)$ forms a system of representatives for all cosets $x H^{\delta}$, $\delta \in S L_{3}(\mathbb{R})$. Since the elements $x$ and $\delta$ can be chosen in the group $\mathrm{SO}_{3}(\mathbb{R})$ we may take $x$ as the matrix

$$
\left(\begin{array}{ccc}
\cos q \cos r-\sin q \sin r \cos p & \cos q \sin r+\sin q \cos r \cos p & \sin q \sin p \\
-\sin q \cos r-\cos q \sin r \cos p & -\sin q \sin r+\cos q \cos r \cos p & \cos q \sin p \\
\sin p \sin r & -\sin p \cos r & \cos p
\end{array}\right)
$$

and $\delta$ as the matrix

$$
\left(\begin{array}{ccc}
\cos \alpha \cos \beta-\sin \alpha \sin \beta \cos \gamma & \cos \alpha \sin \beta+\sin \alpha \cos \beta \cos \gamma & \sin \alpha \sin \gamma \\
-\sin \alpha \cos \beta-\cos \alpha \sin \beta \cos \gamma & -\sin \alpha \sin \beta+\cos \alpha \cos \beta \cos \gamma & \cos \alpha \sin \gamma \\
\sin \gamma \sin \beta & -\sin \gamma \cos \beta & \cos \gamma
\end{array}\right) \text {, }
$$

where $q, r, \alpha, \beta \in[0,2 \pi]$ and $p, \gamma \in[0, \pi]$. The image $\sigma\left(S L_{3}(\mathbb{R}) / H\right)$ forms for all given $\delta \in S O_{3}(\mathbb{R})$ and $x \in S O_{3}(\mathbb{R})$ a system of representatives for 
the cosets $x H^{\delta}$ if and only if there exists unique angles $t, u \in[0,2 \pi]$ and $z \in[0, \pi]$ and unique positive real numbers $a, b$ as well as unique real numbers $k, l, v$ such that the following equation holds

$$
\delta x^{-1} g(t, u, z) f=h \delta,
$$

where the matrices $\delta, x$ have the form as above,

$$
f=\left(\begin{array}{ccc}
f_{1}(t, u, z) & f_{2}(t, u, z) & f_{3}(t, u, z) \\
0 & f_{4}(t, u, z) & f_{5}(t, u, z) \\
0 & 0 & f_{1}^{-1}(t, u, z) f_{4}^{-1}(t, u, z)
\end{array}\right)
$$

and

$$
h=\left(\begin{array}{ccc}
a & k & v \\
0 & b & l \\
0 & 0 & (a b)^{-1}
\end{array}\right)
$$

Comparing the first column of the left and the right side of the equation (12) we obtain the following three equations:

$f_{1}(t, u, z)\{[(\cos \alpha \cos \beta-\sin \alpha \sin \beta \cos \gamma)(\cos r \cos q-\sin r \sin q \cos p)+$ $(\cos \alpha \sin \beta+\sin \alpha \cos \beta \cos \gamma)(\sin r \cos q+\cos r \sin q \cos p)+$ $\sin \alpha \sin \gamma \sin p \sin q](\cos t \cos u-\sin t \sin u \cos z)-$ $[-(\cos \alpha \cos \beta-\sin \alpha \sin \beta \cos \gamma)(\cos r \sin q+\sin r \cos q \cos p)+$ $(\cos \alpha \sin \beta+\sin \alpha \cos \beta \cos \gamma)(-\sin r \sin q+\cos r \cos q \cos p)+$ $\sin \alpha \sin \gamma \sin p \cos q](\sin t \cos u+\cos t \sin u \cos z)+$

$[(\cos \alpha \cos \beta-\sin \alpha \sin \beta \cos \gamma) \sin r \sin p-$ $(\cos \alpha \sin \beta+\sin \alpha \cos \beta \cos \gamma) \cos r \sin p+\sin \alpha \sin \gamma \cos p] \sin z \sin u\}=$ $a(\cos \alpha \cos \beta-\sin \alpha \sin \beta \cos \gamma)-k(\sin \alpha \cos \beta+\cos \alpha \sin \beta \cos \gamma)+$ $v \sin \gamma \sin \beta$,

$f_{1}(t, u, z)\{[-(\sin \alpha \cos \beta+\cos \alpha \sin \beta \cos \gamma)(\cos r \cos q-\sin r \sin q \cos p)-$ $(-\sin \alpha \sin \beta+\cos \alpha \cos \beta \cos \gamma)(\sin r \cos q+\cos r \sin q \cos p)+$ $\cos \alpha \sin \gamma \sin p \sin q](\cos t \cos u-\sin t \sin u \cos z)-$

$[(\sin \alpha \cos \beta+\cos \alpha \sin \beta \cos \gamma)(\cos r \sin q+\sin r \cos q \cos p)+$ $(-\sin \alpha \sin \beta+\cos \alpha \cos \beta \cos \gamma)(-\sin r \sin q+\cos r \cos q \cos p)+$ $\cos \alpha \sin \gamma \sin p \cos q](\sin t \cos u+\cos t \sin u \cos z)+$ $[-(\sin \alpha \cos \beta+\cos \alpha \sin \beta \cos \gamma) \sin r \sin p-(\cos \alpha \cos \beta \cos \gamma-\sin \alpha \sin \beta)$ $\cos r \sin p+\cos \alpha \sin \gamma \cos p] \sin z \sin u\}=$

$-b(\sin \alpha \cos \beta+\cos \alpha \sin \beta \cos \gamma)+l \sin \gamma \sin \beta$,

$f_{1}(t, u, z)\{[(\cos r \cos q-\sin r \sin q \cos p) \sin \gamma \sin \beta-$ $(\sin r \cos q+\cos r \sin q \cos p) \sin \gamma \cos \beta+\cos \gamma \sin p \sin q]$ $(\cos t \cos u-\sin t \sin u \cos z)+[(\cos r \sin q+\sin r \cos q \cos p) \sin \gamma \sin \beta+$ $(-\sin r \sin q+\cos r \cos q \cos p) \sin \gamma \cos \beta+\cos \gamma \sin p \cos q]$ 
$(\sin t \cos u+\cos t \sin u \cos z)+$

$[(\sin \gamma \sin \beta \sin r \sin p+\sin \gamma \cos \beta \cos r \sin p)+\cos \gamma \cos p] \sin z \sin u\}=$ $(a b)^{-1} \sin \gamma \sin \beta$.

If we take $\sin \gamma \sin \beta$ and $\cos \gamma$ as independent variables the third equation turns to the following equations

$$
\begin{aligned}
0= & f_{1}(t, u, z)[\sin p \sin q(\cos t \cos u-\sin t \sin u \cos z)- \\
& \sin p \cos q(\sin t \cos u+\cos t \sin u \cos z)+\cos p \sin z \sin u] \\
(a b)^{-1}= & \{[(\cos r \cos q-\sin r \sin q \cos p)(\cos t \cos u-\sin t \sin u \cos z)+ \\
& (\cos r \sin q+\sin r \cos q \cos p)(\sin t \cos u+\cos t \sin u \cos z)+ \\
& \sin r \sin p \sin z \sin u]- \\
& \frac{\cos \beta}{\sin \beta}[(\sin r \cos q+\cos r \sin q \cos p)(\cos t \cos u-\sin t \sin u \cos z)- \\
& (-\sin r \sin q+\cos r \cos q \cos p)(\sin t \cos u+\cos t \sin u \cos z)- \\
& \cos r \sin p \sin z \sin u]\} f_{1}(t, u, z) .
\end{aligned}
$$

If we take $\cos \alpha \sin \beta \cos \gamma, \sin \beta \sin \gamma$ as independent variables from the second equation it follows

$$
\begin{aligned}
l= & \frac{\cos \alpha}{\sin \beta} f_{1}(t, u, z)[\sin p \sin q(\cos t \cos u-\sin t \sin u \cos z)- \\
& \sin p \cos q(\sin t \cos u+\cos t \sin u \cos z)+\cos p \sin z \sin u] \\
-b= & \{[-(\cos r \cos q-\sin r \sin q \cos p)(\cos t \cos u-\sin t \sin u \cos z)- \\
& (\cos r \sin q+\sin r \cos q \cos p)(\sin t \cos u+\cos t \sin u \cos z)- \\
& \sin r \sin p \sin z \sin u]- \\
& \frac{\cos \beta}{\sin \beta}[(\sin r \cos q+\cos r \sin q \cos p)(\cos t \cos u-\sin t \sin u \cos z)- \\
& (-\sin r \sin q+\cos r \cos q \cos p)(\sin t \cos u+\cos t \sin u \cos z)- \\
& \cos r \sin p \sin z \sin u]\} f_{1}(t, u, z) .
\end{aligned}
$$

If we choose $\sin \alpha \sin \beta \cos \gamma, \sin \beta \sin \gamma$ as independent variables the first equation yields

$$
\begin{aligned}
v= & \frac{\sin \alpha}{\sin \beta} f_{1}(t, u, z)[\sin p \sin q(\cos t \cos u-\sin t \sin u \cos z)- \\
& \sin p \cos q(\sin t \cos u+\cos t \sin u \cos z)+\cos p \sin z \sin u]
\end{aligned}
$$

$$
\begin{aligned}
a+k \frac{\cos \alpha}{\sin \alpha}= & \{[(\cos r \cos q-\sin r \sin q \cos p)(\cos t \cos u-\sin t \sin u \cos z)- \\
& (\cos r \sin q+\sin r \cos q \cos p)(\sin t \cos u+\cos t \sin u \cos z)+ \\
& \sin r \sin p \sin z \sin u]- \\
& \frac{\cos \beta}{\sin \beta}[(\sin r \cos q+\cos r \sin q \cos p)(\cos t \cos u-\sin t \sin u \cos z)- \\
& (-\sin r \sin q+\cos r \cos q \cos p)(\sin t \cos u+\cos t \sin u \cos z)- \\
& \cos r \sin p \sin z \sin u]\} f_{1}(t, u, z) .
\end{aligned}
$$


Since $f_{1}(t, u, z)>0$ from equation (13) it follows that

$$
\begin{aligned}
0= & \sin p \sin q(\cos t \cos u-\sin t \sin u \cos z)+ \\
& \sin p \cos q(\sin t \cos u+\cos t \sin u \cos z)+\cos p \sin z \sin u
\end{aligned}
$$

Using this it follows from (15) that $l=0$ holds and from equation (17) that $v=0$. Since the equation (14) must be satisfied for all $\beta \in[0,2 \pi]$ we have

$$
\begin{aligned}
(a b)^{-1}= & {[(\cos r \cos q-\sin r \sin q \cos p)(\cos t \cos u-\sin t \sin u \cos z)+} \\
& (\cos r \sin q+\sin r \cos q \cos p)(\sin t \cos u+\cos t \sin u \cos z)+ \\
& \sin r \sin p \sin z \sin u] f_{1}(t, u, z) \\
0= & {[(\sin r \cos q+\cos r \sin q \cos p)(\cos t \cos u-\sin t \sin u \cos z)-} \\
& (-\sin r \sin q+\cos r \cos q \cos p)(\sin t \cos u+\cos t \sin u \cos z)- \\
& (\cos r \sin p \sin z \sin u] .
\end{aligned}
$$

Using equation (21) and comparing the equations (20) and (16) we obtain that $(a b)^{-1}=b$. With equation (21) the equation (18) turns to

$$
\begin{aligned}
a+k \frac{\cos \alpha}{\sin \alpha}= & {[(\cos r \cos q-\sin r \sin q \cos p)(\cos t \cos u-\sin t \sin u \cos z)-} \\
& (\cos r \sin q+\sin r \cos q \cos p)(\sin t \cos u+\cos t \sin u \cos z)+ \\
& \sin r \sin p \sin z \sin u] f_{1}(t, u, z) .
\end{aligned}
$$

Since the equation (22) must be satisfied for all $\alpha \in[0,2 \pi]$ we obtain $k=0$. Using this, the equations $(22)$ and $(20)$ yield $(a b)^{-1}=a$. Since $1=a b(a b)^{-1}=a^{3}$ it follows that $a=1$ and hence the matrix $h$ is the identity. But then the matrix equation (12) turns to the matrix equation

$$
g(t, u, z) f=x .
$$

As $x$ and $g(t, u, z)$ are elements of $S O_{3}(\mathbb{R})$ one has $f=x g^{-1}(t, u, z) \in$ $S_{3}(\mathbb{R})$. But then $f$ is the identity, which means that

$$
f_{1}(t, u, z)=1=f_{4}(t, u, z), \quad f_{2}(t, u, z)=f_{3}(t, u, z)=f_{5}(t, u, z)=0,
$$

for all $t, u \in[0,2 \pi]$ and $z \in[0, \pi]$. Since the loop $L$ is isomorphic to the loop $L(\sigma)$ and $L(\sigma) \cong S O_{3}(\mathbb{R})$ there is no connected almost topological proper loop $L$ homeomorphic to $\mathcal{P}_{3}$ such that the group topologically generated by its left translations is isomorphic to $S L_{3}(\mathbb{R})$.

Now we assume that there is an almost topological loop $L$ homeomorphic to $\mathcal{S}_{3}$ such that the group $G$ topologically generated by its left translations is isomorphic to the universal covering group $\widetilde{S L_{3}(\mathbb{R})}$. Then the stabilizer $H$ of the identity of $L$ may be chosen as the group (10). Then there exists a local section $\sigma: U / H \rightarrow G$, where $U$ is a suitable neighbourhood of $H$ in $G / H$ which has the shape (11) with sufficiently small $t, u \in[0,2 \pi], z \in[0, \pi]$ and continuous functions $f_{i}(t, u, z)$ : 
$[0,2 \pi] \times[0,2 \pi] \times[0, \pi] \rightarrow \mathbb{R}$ satisfying the same conditions as there. The image $\sigma(U / H)$ is a local section for the space of the left cosets $\left\{x H^{\delta} ; x \in G, \delta \in G\right\}$ precisely if for all suitable matrices $x:=g(q, r, p)$ with sufficiently small $(q, r, p) \in[0,2 \pi] \times[0,2 \pi] \times[0, \pi]$ there exist a unique element $g(t, u, z) \in \operatorname{Spin}_{3}(\mathbb{R})$ with sufficiently small $(t, u, z) \in$ $[0,2 \pi] \times[0,2 \pi] \times[0, \pi]$ and unique positive real numbers $a, b$ as well as unique real numbers $k, l, v$ such that the matrix equation (12) holds. Then we see as in the case of the group $S L_{3}(\mathbb{R})$ that for small $x$ and $g(t, u, z)$ the matrix $f$ is the identity. Therefore any subloop $T$ of $L$ which is homeomorphic to $\mathcal{S}_{1}$ is locally commutative. Then according to [8], Corollary 18.19, p. 248, each subloop $T$ is isomorphic to a 1dimensional torus group. It follows that the restriction of the matrix $f$ to $T$ is the identity. Since $L$ is covered by such 1-dimensional tori the matrix $f$ is the identity for all elements of $\mathcal{S}_{3}$. Hence there is no proper loop $L$ homeomorphic to $\mathcal{S}_{3}$ such that the group $G$ topologically generated by its left translations is isomorphic to the universal covering group

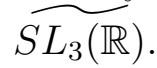

\section{Compact loops with compact-free inner mapping groups}

Proposition 3. Let $L$ be an almost topological loop homeomorphic to a compact connected Lie group $K$. Then the group $G$ topologically generated by the left translations of $L$ cannot be isomorphic to a split extension of a solvable group $R$ homeomorphic to $\mathbb{R}^{n}(n \geq 1)$ by the group $K$.

Proof. Denote by $H$ the stabilizer of the identity of $L$ in $G$. If $G$ has the structure as in the assertion then the elements of $G$ can be represented by the pairs $(k, r)$ with $k \in K$ and $r \in R$. Since $L$ is homeomorphic to $K$ the loop $L$ is isomorphic to the loop $L(\sigma)$ given by a sharply transitive section $\sigma: G / H \rightarrow G$ the image of which is the set $\mathfrak{S}=\{(k, f(k)) ; k \in K\}$, where $f$ is a continuous function from $K$ into $R$ with $f(1)=1 \in R$. The multiplication of $(L(\sigma), *)$ on $\mathfrak{S}$ is given by $(x, f(x)) *(y, f(y))=$ $\sigma((x y, f(x) f(y)) H)$.

Let $T$ be a 1-dimensional torus of $K$. Then the set $\{(t, f(t)) ; t \in T\}$ topologically generates a compact subloop $\tilde{T}$ of $L(\sigma)$ such that the group topologically generated by its left translations has the shape $T U$ with $T \cap U=1$, where $U$ is a normal solvable subgroup of $T U$ homeomorphic to $\mathbb{R}^{n}$ for some $n \geq 1$. The multiplication $*$ in the subloop $\tilde{T}$ is given by

$$
(x, f(x)) *(y, f(y))=\sigma((x y, f(x) f(y)) H)=(x y, f(x y)),
$$

where $x, y \in T$. Hence $\tilde{T}$ is a subloop homeomorphic to a 1 -sphere which has a solvable Lie group $S$ as the group topologically generated by the 
left translations. It follows that $\tilde{T}$ is a 1-dimensional torus group since otherwise the group $S$ would be not solvable (cf. 8], Proposition 18.2, p. 235). As $f: \tilde{T} \rightarrow U$ is a homomorphism and $U$ is homeomorphic to $\mathbb{R}^{n}$ it follows that the restriction of $f$ to $\tilde{T}$ is the constant function $f(\tilde{T})=1$. Since the exponential map of a compact group is surjective any element of $K$ is contained in a one-parameter subgroup of $K$. It follows $f(K)=1$ and $L$ is the group $K$ which is a contradiction.

Theorem 4. Let $L$ be an almost topological proper loop homeomorphic to a sphere or to a real projective space. If the group $G$ topologically generated by the left translations of $L$ is a Lie group and the stabilizer $H$ of the identity of $L$ in $G$ is a compact-free subgroup of $G$, then $L$ is homeomorphic to the 1-sphere and $G$ is a finite covering of the group $P S L_{2}(\mathbb{R})$.

Proof. If $\operatorname{dim} L=1$ then according to Brouwer's theorem (cf. [11], 96.30, p. 639) the transitive group $G$ on $S_{1}$ is a finite covering of $P S L_{2}(\mathbb{R})$.

Now let $\operatorname{dim} L>1$. Since the universal covering of the $n$-dimensional real projective space is the $n$-sphere $\mathcal{S}_{n}$ we may assume that $L$ is homeomorphic to $\mathcal{S}_{n}, n \geq 2$. Since $L$ is a multiplication with identity $e$ on $S_{n}$ one has $n \in\{3,7\}$ (cf. [1]).

Any maximal compact subgroup $K$ of $G$ acts transitively on $L$ (cf. [11], 96.19, p. 636). As $H \cap K=\{1\}$ the group $K$ operates sharply transitively on $L$. Since there is no compact group acting sharply transitively on the 7-sphere (cf. [11], 96.21, p. 637), the loop $L$ is homeomorphic to the 3 -sphere. The only compact group homeomorphic to the 3 -sphere is the unitary group $S U_{2}(\mathbb{C})$. If the group $G$ were not simple, then $G$ would be a semidirect product of the at most 3-dimensional solvable radical $R$ with the group $S U_{2}(\mathbb{C})$ (cf. [4], p. 187 and Theorem 2.1, p. 180). But according to Proposition 3 such a group cannot be the group topologically generated by the left translations of $L$. Hence $G$ is a non-compact Lie group the Lie algebra of which is simple. But then $G$ is isomorphic either to the group $S L_{2}(\mathbb{C})$ or to the universal covering of the group $S L_{3}(\mathbb{R})$. It follows from Proposition 1 and 2 that no of these groups can be the group topologically generated by the left translations of an almost topological proper loop $L$.

\section{The classification of 1 -dimensional compact connected $\mathcal{C}^{1}$-loops}

If $L$ is a connected strongly almost topological 1-dimensional compact loop, then $L$ is homeomorphic to the 1 -sphere and the group topologically generated by its left translations is a finite covering of the group $P S L_{2}(\mathbb{R})$ 
(cf. Proposition 18.2 in [8], p. 235). We want to classify explicitly all 1dimensional $\mathcal{C}^{1}$-differentiable compact connected loops which have either the group $P S L_{2}(\mathbb{R})$ or $S L_{2}(\mathbb{R})$ as the group topologically generated by the left translations.

First we classify the 1-dimensional compact connected loops having $G=S L_{2}(\mathbb{R})$ as the group topologically generated by their left translations. Since the stabilizer $H$ is compact free and may be chosen as the group of upper triangular matrices (see Theorem 1.11, in [8], p. 21) this is equivalent to the classification of all loops $L(\sigma)$ belonging to the sharply transitive $\mathcal{C}^{1}$-differentiable sections

$$
\begin{aligned}
\sigma: & \left(\begin{array}{rr}
\cos t & \sin t \\
-\sin t & \cos t
\end{array}\right)\left\{\left(\begin{array}{cc}
a & b \\
0 & a^{-1}
\end{array}\right) ; a>0, b \in \mathbb{R}\right\} \rightarrow \\
& \left(\begin{array}{rr}
\cos t & \sin t \\
-\sin t & \cos t
\end{array}\right)\left(\begin{array}{cc}
f(t) & g(t) \\
0 & f^{-1}(t)
\end{array}\right) \text { with } t \in \mathbb{R} .
\end{aligned}
$$

Definition 1. Let $\mathcal{F}$ be the set of series

$$
a_{0}+\sum_{k=1}^{\infty}\left(a_{k} \cos k t+b_{k} \sin k t\right), \quad t \in \mathbb{R},
$$

such that

$$
\begin{gathered}
1-a_{0}=\sum_{k=1}^{\infty} \frac{a_{k}+k b_{k}}{1+k^{2}} \\
a_{0}>\sum_{k=1}^{\infty} \frac{k a_{k}-b_{k}}{1+k^{2}} \sin k t-\frac{a_{k}+k b_{k}}{1+k^{2}} \cos k t \text { for all } t \in[0,2 \pi] \\
2 a_{0} \geq \sum_{k=1}^{\infty}\left(a_{k}^{2}+b_{k}^{2}\right) \frac{k^{2}-1}{k^{2}+1} .
\end{gathered}
$$

Lemma 5. The set $\mathcal{F}$ consists of Fourier series of continuous functions.

Proof. Since $\sum_{k=2}^{\infty} a_{k}^{2}+b_{k}^{2}<\frac{10}{3} a_{0}$ it follows from [14], p. 4, that any series in $\mathcal{F}$ converges uniformly to a continuous function $f$ and hence it is the Fourier series of $f$ (cf. [14], Theorem 6.3, p. 12).

Let $\sigma$ be a sharply transitive section of the shape (23). Then $f(t), g(t)$ are periodic continuously differentiable functions $\mathbb{R} \rightarrow \mathbb{R}$, such that $f(t)$ is strictly positive with $f(2 k \pi)=1$ and $g(2 k \pi)=0$ for all $k \in \mathbb{Z}$. 
As $\sigma$ is sharply transitive the image $\sigma(G / H)$ forms a system of representatives for the cosets $x H^{\rho}$ for all $\rho \in G$ (cf. [3]). All conjugate groups $H^{\rho}$ can be already obtained if $\rho$ is an element of $K=$ $\left\{\left(\begin{array}{rr}\cos t & \sin t \\ -\sin t & \cos t\end{array}\right), t \in \mathbb{R}\right\}$. Since $K^{\kappa} H^{\kappa}=K H^{\kappa}$ for any $\kappa \in K$ the group $K$ forms a system of representatives for the left cosets $x H^{\kappa}$.

We want to determine the left coset $x(t) H^{\kappa}$ containing the element

$$
\varphi(t)=\left(\begin{array}{rr}
\cos t & \sin t \\
-\sin t & \cos t
\end{array}\right)\left(\begin{array}{cc}
f(t) & g(t) \\
0 & f^{-1}(t)
\end{array}\right)
$$

where $\kappa=\left(\begin{array}{rr}\cos \beta & \sin \beta \\ -\sin \beta & \cos \beta\end{array}\right)$ and $x(t)=\left(\begin{array}{rr}\cos \eta(t) & \sin \eta(t) \\ -\sin \eta(t) & \cos \eta(t)\end{array}\right)$. The element $\varphi(t)$ lies in the left coset $x(t) H^{\kappa}$ if and only if $\varphi(t)^{\kappa^{-1}} \in$ $x(t)^{\kappa^{-1}} H=x(t) H$. Hence we have to solve the following matrix equation

$$
\begin{gathered}
\left(\begin{array}{rr}
\cos t & \sin t \\
-\sin t & \cos t
\end{array}\right)\left[\kappa\left(\begin{array}{cc}
f(t) & g(t) \\
0 & f^{-1}(t)
\end{array}\right) \kappa^{-1}\right] \\
\left(\begin{array}{rr}
\cos \eta(t) & \sin \eta(t) \\
-\sin \eta(t) & \cos \eta(t)
\end{array}\right)\left(\begin{array}{ll}
a & b \\
0 & a^{-1}
\end{array}\right)
\end{gathered}
$$

for suitable $a>0, b \in \mathbb{R}$. Comparing both sides of the matrix equation (24) we have

$$
\begin{gathered}
f(t) \cos \beta(\sin t \cos \beta-\cos t \sin \beta)-g(t) \sin \beta(\sin t \cos \beta-\cos t \sin \beta)+ \\
f(t)^{-1} \sin \beta(\sin t \sin \beta+\cos t \cos \beta)=\sin \eta(t) a
\end{gathered}
$$

and

$$
\begin{gathered}
f(t) \cos \beta(\cos t \cos \beta+\sin t \sin \beta)-g(t) \sin \beta(\cos t \cos \beta+\sin t \sin \beta)+ \\
f(t)^{-1} \sin \beta(\cos t \sin \beta-\sin t \cos \beta)=\cos \eta(t) a .
\end{gathered}
$$

From this it follows

$$
\tan \eta_{\beta}(t)=\frac{(f(t)-g(t) \tan \beta)(\tan t-\tan \beta)+f^{-1}(t) \tan \beta(1+\tan t \tan \beta)}{(f(t)-g(t) \tan \beta)(1+\tan t \tan \beta)+f^{-1}(t) \tan \beta(\tan \beta-\tan t)} .
$$

Since $\beta$ can be chosen in the intervall $0 \leq \beta<\frac{\pi}{2}$ and $\frac{\pi}{2}<\beta<\pi$ we may replace the parameter $\tan \beta$ by any $w \in \mathbb{R}$.

A $\mathcal{C}^{1}$-differentiable loop $L$ corresponding to $\sigma$ exists if and only if the function $t \mapsto \eta_{w}(t)$ is strictly increasing, i.e. if $\eta_{w}^{\prime}(t)>0$ (cf. Proposition 18.3 , p. 238, in [8]). The function $a_{w}(t): t \mapsto \tan \eta_{w}(t): \mathbb{R} \rightarrow \mathbb{R} \cup\{ \pm \infty\}$ is strictly increasing if and only if $\eta_{w}^{\prime}(t)>0$ since

$$
\frac{d}{d t} \tan \left(\eta_{w}(t)\right)=\frac{1}{\cos ^{2}\left(\eta_{w}(t)\right)} \eta_{w}^{\prime}(t)
$$


A straightforward calculation shows that

$$
\begin{aligned}
\frac{d}{d t} \tan \left(\eta_{w}(t)\right)= & \frac{w^{2}+1}{\cos ^{2}(t)}\left[w^{2}\left(g^{\prime}(t) f(t)+g(t) f^{\prime}(t)+g^{2}(t) f^{2}(t)+1\right)+\right. \\
& \left.w\left(-2 f(t) f^{\prime}(t)-2 g(t) f^{3}(t)\right)+f^{4}(t)\right]
\end{aligned}
$$

Hence the loop $L(\sigma)$ exists if and only if for all $w \in \mathbb{R}$ the inequality

$$
\begin{aligned}
0< & w^{2}\left(g^{\prime}(t) f(t)+g(t) f^{\prime}(t)+g^{2}(t) f^{2}(t)+1\right)+ \\
& w\left(-2 f(t) f^{\prime}(t)-2 g(t) f^{3}(t)\right)+f^{4}(t)
\end{aligned}
$$

holds. For $w=0$ the expression (26) equals to $f^{4}(t)>0$. Therefore the inequality (26) satisfies for all $w \in \mathbb{R}$ if and only if one has

$f^{\prime 2}(t)+g(t) f^{2}(t) f^{\prime}(t)-g^{\prime}(t) f^{3}(t)-f^{2}(t)<0 \quad$ and $\quad g^{\prime}(0)>f^{\prime 2}(0)-1$

for all $t \in \mathbb{R}$. Putting $f(t)=\hat{f}^{-1}(t)$ and $g(t)=-\hat{g}(t)$ these conditions are equivalent to the conditions

$$
\hat{f}^{\prime 2}(t)+\hat{g}(t) \hat{f}^{\prime}(t)+\hat{g}^{\prime}(t) \hat{f}(t)-\hat{f}^{2}(t)<0 \quad \text { and } \quad \hat{g}^{\prime}(0)<1-\hat{f}^{\prime 2}(0)
$$

(cf. 8], Section 18, (C), p. 238).

Now we treat the differential inequality (28). The solution $h(t)$ of the linear differential equation

$$
h^{\prime}(t)+h(t) \frac{\hat{f}^{\prime}(t)}{\hat{f}(t)}+\frac{\hat{f}^{\prime 2}(t)}{\hat{f}(t)}-\hat{f}(t)=0
$$

with the initial conditions $h(0)=0$ and $h^{\prime}(0)=1-\hat{f}^{\prime 2}(0)$ is given by

$$
h(t)=\hat{f}(t)^{-1} \int_{0}^{t}\left(\hat{f}^{2}(t)-\hat{f}^{\prime 2}(t)\right) d t .
$$

Since $\hat{g}(0)=h(0)=0$ and $\hat{g}^{\prime}(0)<h^{\prime}(0)$ it follows from VI in [13 (p. 66) that $\hat{g}(t)$ is a subfunction of the differential equation (29), i.e. that $\hat{g}(t)$ satisfies the differential inequality (28). Moreover, according to Theorem $\mathrm{V}$ in [13] (p. 65) one has $\hat{g}(t)<h(t)$ for all $t \in(0,2 \pi)$. Since the functions $\hat{g}(t)$ and $h(t)$ are continuous $0=\hat{g}(2 \pi) \leq h(2 \pi)$. This yields the following integral inequality

$$
\int_{0}^{2 \pi}\left(\hat{f}^{2}(t)-\hat{f}^{\prime 2}(t)\right) d t \geq 0 .
$$


We consider the real function $R(t)$ defined by $R(t)=\hat{f}(t)-\hat{f}^{\prime}(t)$. Since $\hat{f}(0)=\hat{f}(2 \pi)=1$ and $\hat{f}^{\prime}(0)=\hat{f}^{\prime}(2 \pi)$ we have $R(0)=1-\hat{f}^{\prime}(0)=$ $1-\hat{f}^{\prime}(2 \pi)=R(2 \pi)$.

The linear differential equation

$$
y^{\prime}(t)-y(t)+R(t)=0 \quad \text { with } \quad y(0)=1
$$

has the solution

$$
y(t)=e^{t}\left(1-\int_{0}^{t} R(u) e^{-u} d u\right) .
$$

This solution is unique (cf. [6], p. 2) and hence it is the function $\hat{f}(t)$. The condition $\hat{f}(2 \pi)=1$ is satisfied if and only if $\int_{0}^{2 \pi} R(u) e^{-u} d u=1-\frac{1}{e^{2 \pi}}$. Since $R(t)$ has periode $2 \pi$ its Fourier series is given by

$$
a_{0}+\sum_{k=1}^{\infty}\left(a_{k} \cos k t+b_{k} \sin k t\right)
$$

where $a_{0}=\frac{1}{\pi} \int_{0}^{2 \pi} R(t) d t, a_{k}=\frac{1}{\pi} \int_{0}^{2 \pi} R(t) \cos k t d t$, and $b_{k}=\frac{1}{\pi} \int_{0}^{2 \pi} R(t) \sin k t d t$.

Partial integration yields

$$
\begin{aligned}
& \int_{0}^{t} \sin k u e^{-u} d u=\frac{k-k \cos k t e^{-t}-\sin k t e^{-t}}{1+k^{2}} \\
& \int_{0}^{t} \cos k u e^{-u} d u=\frac{1+k \sin k t e^{-t}-\cos k t e^{-t}}{1+k^{2}} .
\end{aligned}
$$

Using (34) and (35), we obtain by partial integration

$$
\begin{gathered}
\int_{0}^{t} R(u) e^{-u} d u=a_{0}-a_{0} e^{-t}+\sum_{k=1}^{\infty}\left[\int_{0}^{t} a_{k} \cos k u e^{-u} d u+\int_{0}^{t} b_{k} \sin k u e^{-u} d u\right]= \\
a_{0}-a_{0} e^{-t}+\sum_{k=1}^{\infty} \frac{a_{k}\left(1+k \sin k t e^{-t}-\cos k t e^{-t}\right)}{1+k^{2}}+\frac{b_{k}\left(k-k \cos k t e^{-t}-\sin k t e^{-t}\right)}{1+k^{2}} .
\end{gathered}
$$

Now for the real coefficients $a_{0}, a_{k}, b_{k}(k \geq 1)$ it follows

$$
1-\frac{1}{e^{2 \pi}}=\int_{0}^{2 \pi} R(u) e^{-u} d u=\left(a_{0}+\sum_{k=1}^{\infty} \frac{a_{k}+k b_{k}}{1+k^{2}}\right)\left(1-\frac{1}{e^{2 \pi}}\right) .
$$


Hence one has

$$
a_{0}+\sum_{k=1}^{\infty} \frac{a_{k}+k b_{k}}{1+k^{2}}=1
$$

The function $\hat{f}(t)$ is positive if and only if

$$
1>\int_{0}^{t} R(u) e^{-u} d u \text { for all } t \in[0,2 \pi]
$$

Applying (34) and (35) again we see that the inequality (38) is equivalent to

$$
a_{0}>\sum_{k=1}^{\infty}\left[\frac{a_{k} k-b_{k}}{1+k^{2}} \sin k t-\frac{a_{k}+b_{k} k}{1+k^{2}} \cos k t\right] .
$$

Since $\hat{f}^{\prime}(t)+\hat{f}(t)=2 e^{t}\left(1-\int_{0}^{t} R(u) e^{-u} d u\right)-R(t)$ the function $\hat{f}(t)$ satisfies the integral inequality (30) if and only if

$$
\int_{0}^{2 \pi} R(t)\left[2 e^{t}\left(1-\int_{0}^{t} R(u) e^{-u} d u\right)-R(t)\right] d t \geq 0 .
$$

The left side of (40) can be written as

$$
2 \int_{0}^{2 \pi} R(t) e^{t} d t-2 \int_{0}^{2 \pi} R(t) e^{t}\left(\int_{0}^{t} R(u) e^{-u} d u\right) d t-\int_{0}^{2 \pi} R^{2}(t) d t .
$$

Using partial integration and representing $R(u)$ by a Fourier series (33) we have

$$
\int_{0}^{2 \pi} R(t) e^{t} d t=\left(a_{0}+\sum_{k=1}^{\infty} \frac{a_{k}-b_{k} k}{1+k^{2}}\right)\left(e^{2 \pi}-1\right) .
$$

From (36) it follows

$$
\begin{gathered}
\int_{0}^{2 \pi} R(t) e^{t}\left(\int_{0}^{t} R(u) e^{-u} d u\right) d t= \\
a_{0} \int_{0}^{2 \pi} R(t) e^{t} d t-a_{0} \int_{0}^{2 \pi} R(t) d t+\sum_{k=1}^{\infty} \int_{0}^{2 \pi}\left(\frac{a_{k}+k b_{k}}{1+k^{2}}\right) R(t) e^{t} d t+
\end{gathered}
$$




$$
\sum_{k=1}^{\infty} \int_{0}^{2 \pi}\left(\frac{k a_{k}-b_{k}}{1+k^{2}}\right) R(t) \sin k t d t-\sum_{k=1}^{\infty} \int_{0}^{2 \pi}\left(\frac{a_{k}+k b_{k}}{1+k^{2}}\right) R(t) \cos k t d t
$$

Substituting for $R(t)$ its Fourier series and applying the relation (a) in [12] (p. 10) we have

$$
\int_{0}^{2 \pi} R(t) d t=2 \pi a_{0}
$$

Futhermore, one has

$$
\begin{gathered}
\sum_{k=1}^{\infty} \int_{0}^{2 \pi}\left(\frac{k a_{k}-b_{k}}{1+k^{2}}\right) R(t) \sin k t d t= \\
\sum_{k=1}^{\infty} \int_{0}^{2 \pi}\left(\frac{k a_{k}-b_{k}}{1+k^{2}}\right)\left[a_{0}+\sum_{l=1}^{\infty}\left(a_{l} \cos l t+b_{l} \sin l t\right)\right] \sin k t d t= \\
a_{0} \sum_{k=1}^{\infty} \int_{0}^{2 \pi}\left(\frac{k a_{k}-b_{k}}{1+k^{2}}\right) \sin k t d t+\sum_{k=1}^{\infty} \sum_{l=1}^{\infty} \int_{0}^{2 \pi}\left(\frac{k a_{k}-b_{k}}{1+k^{2}}\right) a_{l} \cos l t \sin k t d t+ \\
\sum_{k=1}^{\infty} \sum_{l=1}^{\infty} \int_{0}^{2 \pi}\left(\frac{k a_{k}-b_{k}}{1+k^{2}}\right) b_{l} \sin l t \sin k t d t
\end{gathered}
$$

The relations (a), (b), (c), (d) in [12], p. 10, yield

$$
\sum_{k=1}^{\infty} \int_{0}^{2 \pi}\left(\frac{k a_{k}-b_{k}}{1+k^{2}}\right) R(t) \sin k t d t=\sum_{k=1}^{\infty} \int_{0}^{2 \pi}\left(\frac{k a_{k}-b_{k}}{1+k^{2}}\right) b_{k} \sin ^{2} k t d t=\sum_{k=1}^{\infty}\left(\frac{k a_{k}-b_{k}}{1+k^{2}}\right) b_{k} \pi .
$$

Analogously we obtain that

$$
\sum_{k=1}^{\infty} \int_{0}^{2 \pi}\left(\frac{a_{k}+k b_{k}}{1+k^{2}}\right) R(t) \cos k t d t=\sum_{k=1}^{\infty} \int_{0}^{2 \pi}\left(\frac{k a_{k}+b_{k}}{1+k^{2}}\right) b_{k} \cos ^{2} k t d t=\sum_{k=1}^{\infty}\left(\frac{a_{k}+k b_{k}}{1+k^{2}}\right) a_{k} \pi .
$$

Using the equality (37) one has

$$
\begin{gathered}
\int_{0}^{2 \pi} R(t) e^{t}\left(\int_{0}^{t} R(u) e^{-u} d u\right) d t= \\
{\left[a_{0}+\sum_{k=1}^{\infty} \frac{a_{k}-k b_{k}}{1+k^{2}}\right]\left(e^{2 \pi}-1\right)-\pi \sum_{k=1}^{\infty} \frac{b_{k}^{2}+a_{k}^{2}}{1+k^{2}}-2 \pi a_{0}^{2} .}
\end{gathered}
$$


Substituting for $R(t)$ its Fourier series we have

$$
\begin{gathered}
\int_{0}^{2 \pi} R^{2}(t) d t=\int_{0}^{2 \pi} a_{0}^{2} d t+2 a_{0} \sum_{k=1}^{\infty} \int_{0}^{2 \pi}\left(a_{k} \cos k t+b_{k} \sin k t\right) d t- \\
\sum_{k=1}^{\infty} \sum_{l=1}^{\infty} \int_{0}^{2 \pi}\left(a_{k} a_{l} \cos k t \cos l t+a_{k} b_{l} \cos k t \sin l t+\right. \\
\left.b_{k} a_{l} \sin k t \cos l t+b_{k} b_{l} \sin k t \sin l t\right) d t .
\end{gathered}
$$

Applying the relations (a), (b), (c), (d) in [12] (p. 10) we obtain

$$
\int_{0}^{2 \pi} R^{2}(t) d t=2 \pi a_{0}^{2}+\pi \sum_{k=1}^{\infty}\left(a_{k}^{2}+b_{k}^{2}\right) .
$$

Hence the integral inequality (30) holds if and only if

$$
2 a_{0} \geq \sum_{k=1}^{\infty}\left(a_{k}^{2}+b_{k}^{2}\right) \frac{k^{2}-1}{k^{2}+1} .
$$

Since the Fourier series of $R(t)$ lies in the set $\mathcal{F}$ of series the Fourier series of $R$ converges uniformly to $R$ (Lemma 5 ).

Summarizing our discussion we obtain the main part of the following

Theorem 6. Let $L$ be a 1-dimensional connected $\mathcal{C}^{1}$-differentiable loop such that the group topologically generated by its left translations is isomorphic to the group $S L_{2}(\mathbb{R})$. Then $L$ is compact and belongs to a $\mathcal{C}^{1}$ differentiable sharply transitive section $\sigma$ of the form

$$
\begin{aligned}
\sigma: & \left(\begin{array}{rr}
\cos t & \sin t \\
-\sin t & \cos t
\end{array}\right)\left\{\left(\begin{array}{ll}
a & b \\
0 & a^{-1}
\end{array}\right) ; a>0, b \in \mathbb{R}\right\} \rightarrow \\
& \left(\begin{array}{rr}
\cos t & \sin t \\
-\sin t & \cos t
\end{array}\right)\left(\begin{array}{cc}
f(t) & g(t) \\
0 & f^{-1}(t)
\end{array}\right) \text { with } t \in \mathbb{R}
\end{aligned}
$$

such that the inverse function $f^{-1}$ has the shape

$$
\begin{gathered}
f^{-1}(t)=e^{t}\left(1-\int_{0}^{t} R(u) e^{-u} d u\right)= \\
a_{0}+\sum_{k=1}^{\infty} \frac{\left(k a_{k}-b_{k}\right) \sin k t+\left(a_{k}+k b_{k}\right) \cos k t}{1+k^{2}}
\end{gathered}
$$


where $R(u)$ is a continuous function the Fourier series of which is contained in the set $\mathcal{F}$ and converges uniformly to $R$, and $g$ is a periodic $\mathcal{C}^{1}$-differentiable function with $g(0)=g(2 \pi)=0$ such that

$$
g(t)>-f(t) \int_{0}^{t} \frac{\left(f^{2}(u)-f^{\prime 2}(u)\right)}{f^{4}(u)} d u \text { for all } t \in(0,2 \pi) .
$$

Conversely, if $R(u)$ is a continuous function the Fourier series of which is contained in $\mathcal{F}$, then the section $\sigma$ of the form (45) belongs to a loop if $f$ is defined by (46) and $g$ is a $\mathcal{C}^{1}$-differentiable periodic function with $g(0)=g(2 \pi)=0$ satisfying $(47)$.

The isomorphism classes of loops defined by $\sigma$ are in one-to-one correspondence to the 2 -sets $\{(f(t), g(t)),(f(-t),-g(-t))\}$.

Proof. The only part of the assertion which has to be discussed is the isomorphism question. It follows from [7], Theorem 3, p. 3, that any isomorphism class of the loops $L$ contains precisely two pairs $\left(f_{1}, g_{1}\right)$ and $\left(f_{2}, g_{2}\right)$. If $\left(f_{1}, g_{1}\right) \neq\left(f_{2}, g_{2}\right)$ and if $\left(f_{1}, g_{1}\right)$ satisfy the inequality $(27)$, then we have

$$
f_{2}^{\prime 2}(-t)+g_{2}(-t) f_{2}^{2}(-t) f_{2}^{\prime}(-t)-g_{2}^{\prime}(-t) f_{2}^{3}(-t)-f_{2}^{2}(-t)<0 .
$$

since from $f_{1}(t)=f_{2}(-t)$ and $g_{1}(t)=-g_{2}(-t)$ we have $f_{1}^{\prime}(t)=-f_{2}^{\prime}(-t)$ and $g_{1}^{\prime}(t)=g_{2}^{\prime}(-t)$.

Remark. A loop $\tilde{L}$ belonging to a section $\sigma$ of shape (45) is a 2-covering of a $\mathcal{C}^{1}$-differentiable loop $L$ having the group $P S L_{2}(\mathbb{R})$ as the group topologically generated by the left translations if and only if for the functions $f$ and $g$ one has $f(\pi)=1$ and $g(\pi)=0$ (cf. [9], p. 5106). Moreover, $L$ is the factor loop $\tilde{L} /\left\{\left(\begin{array}{cc}\epsilon & 0 \\ 0 & \epsilon\end{array}\right) ; \epsilon= \pm 1\right\}$. Any $n$-covering of $L$ is a non-split central extension $\hat{L}$ of the cyclic group of order $n$ by $L$. The loop $\hat{L}$ has the $n$-covering of $P S L_{2}(\mathbb{R})$ as the group topologically generated by its left translations.

\section{References}

[1] Adams JF (1960) On the non-existence of elements of Hopf invariant one. Ann of Math 72: 20-104

[2] Asoh T (1987) On smooth SL(2, C) actions on 3-manifolds. Osaka J Math 24: $271-298$

[3] Baer R (1939) Nets and groups. Trans Amer Math Soc 46: 110-141 
[4] Gorbatsevich VV, Onishchik AL (1993) Lie Transformation Group. In: Onishchik AL (ed) Lie Groups and Lie Algebras I, Encyklopedia of Mathematical Sciences, vol 20, pp 95-229: Berlin Heidelberg New York: Springer

[5] Hofmann KH (1958) Topological Loops. Math Z 70: 125-155

[6] Kamke E (1951) Differentialgleichungen Lösungsmethoden und Lösungen. Mathematik und Ihre Anwendungen in Physik und Technik. Band 181. Leipzig: Akademische Verlagsgesellschaft Geest-Portig K.-G

[7] Nagy PT (2006) Normal form of 1-dimensional differentiable loops. Acta Sci Math 72: 863-873

[8] Nagy PT, Strambach K (2002) Loops in Group Theory and Lie Theory. de Gruyter Expositions in Mathematics 35. Berlin New York: de Gruyter

[9] Nagy PT, Strambach K (2006) Coverings of Topological Loops. Journal of Math Sci 137: 5098-5116

[10] Nagy PT, Stuhl I (2007) Differentiable loops on the real line. Publ Math 70: $361-370$

[11] Salzmann H, Betten D, Grundhöfer T, Hähl H, Löwen R, Stroppel M (1995) Compact Projective Planes. de Gruyter Expositions in Mathematics 21. Berlin New York: de Gruyter

[12] Walker JS (1988) Fourier Analysis. New York Oxford: Oxford University Press

[13] Walter W (1970) Differential and Integral Inequalities. Ergebnisse der Mathematik und ihrer Grenzgebiete. Band 55. Berlin Heidelberg New York: Springer

[14] Zygmund A. (1968) Trigonometric Series. vol I. Cambridge: Cambridge University Press

Author's address: Ágota Figula, Mathematisches Institut der Universität ErlangenNürnberg, Bismarckstr. 1 1/2, 91054 Erlangen, Germany and Institute of Mathematics, University of Debrecen, P.O.B. 12, H-4010 Debrecen, Hungary

e-mail:figula@math.klte.hu

Karl Strambach, Mathematisches Institut der Universität Erlangen-Nürnberg, Bismarckstr. 1 1/2, 91054 Erlangen, Germany e-mail:strambach@mi.uni-erlangen.de 